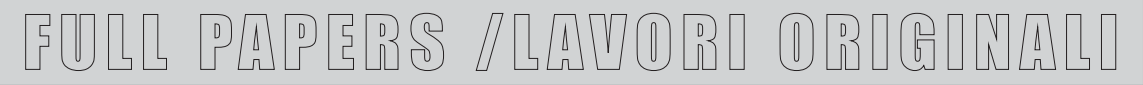

\section{Enteriti acute e protratte nell'uomo: prevalenze eziologiche ed aspetti di metodologia diagnostica}

\author{
Daniele Crotti', Maria Letizia D'Annibale ${ }^{2}$ \\ 'Libero Professionista e Docente in Parassitologia e Microbiologia Medica (già U.O. di Microbiologia, \\ Azienda Ospedaliera, Perugia) \\ U.O. di Microbiologia, Azienda Ospedaliera, Perugia
}

Key words: enteritis, Campylobacter, diagnosis, Rotavirus, Salmonella

Acute and prolonged diarrhoeas in humans: prevalences and diagnosis

\section{SUMMARY}

The Authors describe methodological diagnostic course related to microbiological examination of faeces specimens. During 2004, 5 I I samples of 247 children and 264 adults were analysed for acute (389 subjects) or prolonged diarrhoeas (I 22). Campylobacter was observed in 57 cases ( $11.0 \%$ ), in 36 children (I4.2\%) and 21 adults (8.0\%); Salmonella was observed in 28 cases (5.4\%), in 15 children (6.1\%) and I 3 adults (4.9\%); toxin A of C. difficile was detected in II cases (2.2\%); other bacterial pathogens were very rare. Rotavirus was detected in $3 \mathrm{I}$ children (12.5\%) while Adenovirus in 3 children (1.2\%). Protozoa were identified in I 3 cases (2.5\%): 3 in children (I.2\%) and 10 in adults (3.7\%); among these, D. fragilis prevailed in respect of G. duodenalis: 10 cases $(2.0 \%)$ vs 3 cases $(0.6 \%)$; other protozoa never were observed. So, the positivities were only I5I for $29.5 \%$ ( 9 I in children, $36.8 \%$ and 60 in adults, $22.7 \%$ ). Finally some considerations relating sex, clinical features and epidemiological findings are reported. The Authors reaffirm the need for a rational and functional research, because is necessary to minimize and direct these ones towards local epidemiology, but underline the opportunity to increase the investigations when diversified risk factor are reported.

\section{INTRODUZIONE}

Relativamente numerosi sono gli agenti eziologici responsabili di enterite nell'uomo, siano essi batterici, virali o protozoari $(1,4,21)$.

In Italia sembrano tuttora prevalere Salmonella, tra gli agenti batterici, e Rotavirus, tra quelli virali (sia pur pressoché esclusivamente nella popolazione pediatrica) $(1,16,21)$. Infezioni intestinali sostenute da altri agenti microbici o virali sono meno frequenti, talora rare, se non del tutto assenti o apparentemente tali $(13,19,21,22,24)$.

Unica eccezione, forse, è rappresentata da Campylobacter (nelle sue specie termotolleranti); infatti, se in alcune casistiche sembra essere raro, in altre è per contro frequente, al pari, o quasi, di Salmonella $(5,14)$.

Sicuramente molte infezioni intestinali sono tuttora orfane di agente eziologico specifico $(7,16)$. Questo in parte è conseguenza di scarse attenzioni al problema, soprattutto da un punto di vista di metodologia diagnostica, in parte è conseguenza di difficoltà oggettive concernenti le possibilità diagnostiche medesime (carenza di risorse economiche, gestionali, umane), in parte infine è conseguenza di tuttora probabili misconoscenze di altri agenti eziologici coinvolti in tali patologie infettive $(3,4,17)$.

Indubbiamente esistono variabilità riguardo le prevalenze eziologiche, e in particolar modo variabilità epidemiologiche geografiche (si pensi, in primis, alle cosiddette "diarree del viaggiatore"), ma è altresì vero che altri fattori, virtuali o reali, non di rado intervengono nel determinismo degli agenti eziologici in causa $(4,6,27)$. Tra questi vi sono le popolazioni coinvolte o in studio (se pediatrica o adulta, ad esempio), gli aspetti immunitari relativi ai soggetti stessi (immunocompetenza o immunocompromissione), l'ospedalizzazione (antecedente l'episodio enteritico, evidentemente) o meno, le abitudini alimentari, e così via, tutti fattori o elementi che possono in un qualche modo differenziare non tanto le incidenze quanto le prevalenze delle medesime $(3,4,6,18)$.

In ogni caso spesso l'approccio diagnostico al problema è male impostato, talora è grossolano e superficiale, a volte sottovalutato se non del tutto ignorato, altre volte infine è affrontato in modo riduttivo o comunque insufficiente $(3,4,6)$.

Con tale presentazione vogliamo ribadire l'importanza di un intervento diagnostico metodologi- 
co, ragionato, razionale, al fine di comprendere in modo più concreto le frequenze eziologiche, oltreché le relative prevalenze, delle diarree infettive, siano esse acute (come più sovente accade) o prolungate nel tempo (oltre i 14 giorni), sia nel bambino che nell'adulto, limitandoci peraltro a quelle contratte esclusivamente in ambiente comunitario $(4,6)$.

\section{MATERIALI E METODI}

Nel corso del 2004 sono stati analizzati i campioni fecali di 511 soggetti ( 247 bambini di età compresa tra 1 e 14 anni di vita, pari al $48 \%$ dei soggetti considerati, e 264 adulti, pari al 52\%), per un esame microbiologico mirato in quanto soggetti affetti da enterite (3).

A tutti i campioni pervenuti, rappresentati da feci non formate/semiliquide/liquide, era allegata una specifica scheda clinico-anamnestica, nella quale erano riportate le generalità dei soggetti, i principali aspetti clinici, i richiami anamnestico-epidemiologici essenziali, tali anche da giustificare una richiesta di "coprocoltura", sulla base dell'evidenza o del fondato sospetto di un'enterite in atto, vuoi acuta, vuoi protratta oltre i 14 giorni $(3,4,6,10)$. In tale indagine, a valenza retrospettiva, sono stati esclusi tutti i soggetti per i quali le informazioni fornite erano insufficienti ad inquadrare il caso, tutti i soggetti in terapia antibiotica in atto o cessata da meno dil settimana, tutti i soggetti ospedalizzati nei quali la diarrea era insorta dopo 2 giorni dal ricovero, tutti i controlli e tutti i soggetti con diagnosi diversa da un'enterite vera e propria $(4,6,10)$.

I campioni fecali sono stati tutti spontaneamente raccolti nella stessa mattinata in cui venivano consegnati in laboratorio microbiologico e quindi esaminati, sia fossero di provenienza ambulatoriale, sia fossero di pazienti ricoverati in ambiente nosocomiale specificatamente per tale patologia infettiva intestinale $(3,4,6)$.

Tutti i campioni venivano inizialmente valutati microscopicamente per verificare la qualità e la quantità del materiale fecale, e quindi ritenuti idonei per essere adeguatamente analizzati, nonché per verificare la eventuale presenza di muco e/o sangue evidente (4). Indi venivano osservati al microscopio ottico per rilevare la eventuale presenza di leucociti/fagociti, di sangue occulto, di elementi parassitari evidenti $(4,6)$.

Dopo di che venivano sempre allestite le coprocolture per Salmonella, Shigella e Campylobacter $(3,4,6)$; per Campylobacter venne utilizzata la tecnica della membrana filtrante $(4,8)$. La ricerca di altri patogeni batterici, sempre tramite coprocolture specifiche, venne eseguita in un numero ridotto di casi, sulla base soprattutto delle infor- mazioni desunte dalla scheda $(4,6)$. Per Clostridium difficile si utilizzò una tecnica immunoenzimatica specifica per la ricerca della tossina A dallo stesso prodotta $(15,20)$.

Gli isolati batterici vennero identificati con le consuete procedure diagnostiche, sia biochimiche che immunologiche, come altrove descritto $(4,6)$. Sugli stessi venne sempre poi eseguita la valutazione della sensibilità ai farmaci antibatterici con il sistema di diffusione in agar, secondo quanto già descritto $(4,6,12)$.

Per quanto riguarda gli agenti virali, era possibile esclusivamente la ricerca di Rotavirus ed Adenovirus; questa venne condotta soprattutto in soggetti pediatrici, qualora richiesta ma soprattutto sulla base della sintomatologia clinica (presenza di vomito) e se assenti ematochezia e leucociti, ed eventualmente in quei bambini già risultati negativi ai tre principali agenti batterici sopra riferiti $(6,10,16,21)$. Vennero utilizzati metodi immunologici al lattice (per Rotavirus e Adenovirus) o immunoenzimatici (per il solo Rotavirus) (4, 16, 21).

Per quanto concernette i protozoi, quando ritenuto necessario venne condotta la colorazione permanente di Giemsa (essenzialmente per rilevare o confermare la presenza di Dientamoeba fragilis) e/o la colorazione all'acido resistenza per i coccidi, e di fatto per il rilevamento delle oocisti di Cryptosporidium spp. (1).

\section{RISULTATI}

In tabella 1 vengono riportati i risultati nel loro complesso e discriminando tra soggetti pediatrici ed adulti. Nel corso di tale annata Campylobacter spp. (di fatto C. jejuni/C. coli) prevalse rispetto a Salmonella spp. (Salmonella enterica), con 57 casi $(11.0 \%)$ versus (vs) 28 casi $(5.4 \%)$, sia nei bambini (36 casi vs 15 , pari al $14.2 \%$ vs $6.1 \%$ rispettivamente), sia negli adulti (21 casi vs 13, pari all' $8.0 \%$ vs il $4.9 \%$ ). Altri agenti batterici sono stati isolati in un numero assai limitato di circostanze: vibrioni nell'1\%, Shigella spp. nello $0.4 \%$, C.difficile produttore di tossina A nel $2.2 \%$ dei casi complessivi. Altri batteri patogeni non furono mai reperiti. Per la precisione va detto che all'interno del gruppo di Escherichia coli enteritogeni, si poterono ricercare, e in un numero assai ristretto di circostanze, solamente i ceppi EPEC, ossia quelli agglutinati dai comuni antisieri commerciali, e i ceppi EHEC, ricercati su tutti i campioni di feci con ematochezia. In ogni caso gli esiti furono sempre negativi; altresì Yersinia enterocolitica e Yersinia spp. non fu mai isolata, così come Listeria monocytogenes, ricercata peraltro in pochissime circostanze.

Tra gli agenti virali, Rotavirus è stato osservato in 
31 casi pediatrici (pari al 12.5\%), mentre Adenovirus soltanto in 3 bambini (1.2\%).

Gli agenti protozoari sono stati osservati nel 2.5\% dei casi (13 soggetti complessivi), con maggiore frequenza negli adulti (10 casi, pari al 3.7\%) rispetto ai bambini (3 casi, pari all'1.2\%).

Complessivamente un agente eziologico è stato individuato soltanto nel $29.5 \%$ dei casi (pari a 151 soggetti), in particolare nel $36.8 \%$ dei bambini (91 casi) e $22.7 \%$ (60 casi) tra gli adulti.

In tabella 2 vengono riportate le eziologie nei 389 casi (209 bambini e 180 adulti) di diarree acute (insorte da meno di 10 giorni dalla richiesta della prestazione analitica), mentre in tabella 3 vengono riportate le eziologie nei 122 casi (38 bambini e 84 adulti) di diarree protratte (insorte da oltre 14 giorni dalla richiesta della prestazione analitica). Nel gruppo dei soggetti con diarrea acuta le positività hanno rappresentato il $33.4 \%$ (130 casi, di cui 80 in bambini e 50 in adulti, pari al $38.3 \%$ e $27.8 \%$ rispettivamente), mentre nel gruppo dei soggetti con diarrea protratta le positività hanno rappresentato il $17.2 \%$ (21 casi, di cui 11 in bambini e 10 in adulti, pari al $28.9 \%$ e all' $11.9 \%$ rispettivamente). Nei bambini con diarrea acuta l'agente eziologico più frequente è stato Rotavirus (14.8\%), seguito da Campylobacter (14.4\%) e da Salmonella (5.3\%).

Negli adulti con diarrea acuta Campylobacter è stato isolato nel $10.0 \%$ dei casi mentre Salmonella nel $6.7 \%$.

Nei bambini con diarrea protratta Campylobacter ha leggermente prevalso rispetto a Salmonella $(13.1 \%$ vs $10.5 \%)$, mentre agenti protozoari sono stati osservati nel $5.3 \%$ dei casi.

Negli adulti con diarrea protratta hanno invece prevalso le infezioni protozoarie con il $4.8 \%$ dei casi, rispetto sia a quelle sostenute da Campylobacter $(3.5 \%)$ che a quelle sostenute da Salmonella (1.2\%).

Per quanto concerne il sesso, (tabella 4) va detto che la distribuzione dei casi di Campylobacter, di Salmonella e di Rotavirus è stata più frequente nei soggetti di sesso maschile rispetto a quelli di sesso femminile. Così, 36 su 56 casi di campylobacteriosi sono stati osservati in maschi ( $64.3 \%$ di tutti i casi sostenuti da Campylobacter), 14 su 27 casi di salmonellosi sono stati osservati in maschi (51.8\%) e 21 su 33 casi di infezione da Rotavirus sono stati reperiti nei maschi $(63.6 \%)$. In tale conteggio viene escluso l'unico caso di infezione mista da Campylobacter e Salmonella osservata in un maschio con diarrea protratta. Le infezioni sostenute da protozoi hanno invece prevalso nel sesso femminile, con 8 casi su 13, pari al $61.5 \%$, mentre nei soggetti di sesso maschile sono state osservate nel $38.5 \%$ dei casi. Senza entrare nel merito delle correlazioni tra agente eziologico e aspetti clinici, in quanto non dissimili da quanto riferito in precedenti segnalazioni $(7,10,12,16)$, ci preme soltanto sottolineare ancora una volta la stretta associazione tra presenza di vomito e infezione virale sostenuta da Rotavirus: questa avvenne nel $96.8 \%$ dei casi, laddove il vomito nelle campylobacteriosi era presente soltanto nel $19.3 \%$ dei casi e nelle salmonellosi nel $25.0 \%$ (6, 16). E nel $74.2 \%$ dei casi di infezione intestinale da Rotavirus si rese necessaria una ospedalizzazione dei soggetti, mentre questo accadde nel $64.3 \%$ dei casi sostenuti da Salmonella e nel $26.3 \%$ delle campylobacteriosi. Nessuna specificità infine per altri segni o sintomi, febbre compresa, presente nel $50.0 \%$ delle salmonellosi, nel 41.9\% delle infezioni da Rotavirus, nel 29.8\% delle campylobacteriosi $(7,10,12,16,20)$.

Leucocitosi fecale, assente delle infezioni protozoarie e pressoché assente nelle infezioni da Rotavirus (3.2\%), ha prevalso nelle salmonellosi $(50.0 \%)$ rispetto alle campylobacteriosi $(35.1 \%)$. Altrettanto ematochezia (sempre assente nelle infezioni virali e protozoarie) è stata più frequente, seppur lievemente, nelle infezioni da Salmonella $(25.0 \%)$ rispetto a quelle sostenute da Campylobacter (21.1\%).

Per quanto concerne la stagionalità, 1'83.9\% delle infezioni da Rotavirus si sono osservate tra gennaio e aprile, mentre le infezioni sostenute da Salmonella e Campylobacter si sono osservate un po' sempre nel corso dell'anno, con lieve prevalenza per entrambe tra maggio e agosto $(42.9 \%$ e $43.9 \%$ rispettivamente), come riportato in tabella 5 . Mentre la variabilità delle sottospecie (o biovar) di Salmonella enterica ricalca quanto negli anni passati osservato, con prevalenza di $S$. enterica Typhimurium, per quanto riguarda Campylobacter, nel corso del 2004 ha nettamente prevalso C. jeju$n i$, identificato nel $98 \%$ di tutti i casi di campylobacteriosi, mentre $C$. coli è stato identificato una sola volta, pari al $2 \%(4,5,14,17)$.

Omettiamo volutamente in tale presentazione i patterns di resistenza alle molecole antibiotiche osservati per tutti i ceppi batterici saggiati. Questo in quanto precipua finalità del lavoro era quella di evidenziare la metodologia operativa adottata e relativa alle procedure diagnostiche standard da noi in uso dei campioni di feci, e le correlazioni tra agenti eziologici, caratteristiche cliniche e aspetti epidemiologici, in parte motivanti eventuali ricerche allargate e differenziate $(3,6)$.

\section{DISCUSSIONE}

Quanto riportato non discrimina tra infezioni contratte nella realtà perugina ed umbra più in generale o infezioni contratte altrove, in Paesi a maggiore o diversa endemia (in altri termini "diarrea 
del viaggiatore"). Ad ogni buon conto la stragrande maggioranza delle campylobacteriosi e delle salmonellosi furono contratte "in casa". In soli 4 casi per le prime $(14 \%)$ ed altrettanti 4 per le seconde $(7 \%)$ fu riferito un recente pregresso viaggio all'estero, vuoi in Paesi dell'est europeo o i Paesi africani $(4,27)$. Mai questo fu riferito per le infezioni sostenute da Rotavirus, che pertanto possono a ragione definirsi tutte autoctone. Rotavirus fu essenzialmente ricercato in campo pediatrico. Per altro, in rare circostanze, ed in pazienti riferenti anche vomito (nonché su indicazioni anamnestiche motivate), e risultati negativi per altri più probabili patogeni, tale agente virale fu preso in considerazione. I due casi positivi sono così riferibili ad una giovane adulta insegnante di scuola materna con feci liquide, vomito, dolori addominali e febbre (senza leucocitosi e con ematochezia assente), e ad un anziano che lamentava vomito e diarrea da quattro giorni, senza febbre, e con leucocitosi fecale ed ematochezia assenti.

In rarissimi casi poi fu possibile ipotizzare, riferendosi alle enteriti sostenute dai 3 principali agenti eziologici sopra riportati, la sorgente, se alimentare, dell'infezione; e questo in quanto pur tentando di operare al meglio, spesso sfugge il dato anamnestico relativo proprio al consumo di cibi particolari e a rischio $(4,6)$.

Per quanto riguarda invece i 2 casi di shigellosi (in adulti), entrambe erano espressione di una diarrea del viaggiatore: $S$. sonnei al rientro da un viaggio in Egitto, S. flexneri al rientro da un breve soggiorno alle Isole Maldive. I 3 casi, sempre in soggetti adulti, di infezione da Vibrio spp., riconobbero in parte un'eziologia esotica (1 caso di $V$. cholerae non O1/O139 importato dall'India), ed in parte un'eziologia autoctona dopo consumo di prodotti ittici e di frutti di mare (2 casi di V. parahaemolyticus). Anche i 2 casi sostenuti da Aeromonas spp. furono ritenuti in qualche modo autoctoni: 1 caso di $A$. sobria, in bambina al rientro da una vacanza al Mare Adriatico, ed 1 caso di infezione da $A$. caviae in un paziente adulto immodepresso (in chemioterapia per linfoma non Hodgkin).

Le diarree sostenute da ceppi di $C$. difficile tossino-produttore possono osservarsi, sia pur di rado, anche in soggetti non in terapia antibiotica (in atto, recente o meno recente) e non ospedalizzati, come già nel passato da noi osservato (15). Gli attuali 3 casi pediatrici (1.2\%) e gli 8 casi adulti $(3.0 \%)$ ne sono un evidente esempio (15). Questo a dire che sia nelle diarrea protratte che in quelle acute, le tossine di tale microrganismo anaerobio sporigeno andrebbero ricercate ogniqualvolta la ricerca di altri più comuni patogeni risultasse negativa $(6,15,20)$.
Infine, in tema di protozoosi, gli agenti responsabili furono sempre $D$. fragilis o Giardia duodenalis (Tabella 6). D. fragilis fu identificata nel 2.1\% dei soggetti, G. duodenalis nello $0.6 \%$. La prima fu pertanto nettamente prevalente rispetto alla seconda, prevalse nella popolazione adulta, e sopratutto in soggetti di sesso femminile (7 casi su 10). In un solo caso di giardiasi in un soggetto adulto, infine, venne riferito un pregresso viaggio in un Paese in via di sviluppo tecnologico $(1,4,9)$.

Cryptosporidium e altri coccidi non furono mai repertati, così come protozoi appartenenti al gruppo delle amebe $(1,4)$.

Per concludere da tale semplice presentazione emerge innanzitutto tutto come alcuni patogeni siano con frequenze variabilissime presenti, mentre altri sono del tutto rari, di importazione, o assenti. Tra i patogeni batterici, sicuramente emerge un dato importante, ossia che Campylobacter è stato, nel corso del 2004, più frequente di Salmonella. Al proposito va detto che nel corso di tale periodo non sono stati isolati altre specie di Campylobacter (seppure un ceppo di $C$. jejuni fosse stato identificato come $C$. jejuni sottospecie doylei, e non sottospecie jejuni come in tutti gli altri casi) o ceppi di generi affini, come in passato $(5,8,10,12)$.

L'approccio metodologico è stato sempre quello riportato nelle parti introduttive. Accanto alle osservazioni macro e microscopiche (da cui eventualmente le colorazioni per i protozoi) venivano sempre ricercati, come protocollo minimo, Salmonella, Campylobacter e Shigella; e questo anche in virtù di esperienze passate che individuavano soltanto nei primi due i patogeni presenti nella nostra realtà, quantomeno con prevalenze degne di rilievo $(4,6,10,12,16)$. Shigella, seppur rara e di importazione (in tale casistica), va sempre cercata perché patogeno umano per eccellenza (19). Sulla base poi di altri elementi, clinico-anamnestici ed epidemiologici, le ricerche venivano di volta in volta allargate ad altri patogeni $(6,23,25)$. Infine, la ricerca di Rotavirus, ci sembra obbligatoria nei soggetti, in particola modo bambini, con vomito, ospedalizzati o meno, qualora all'esame diretto non risultasse la presenza di sangue e/o leucociti $(16,21)$.

Sicuramente altri patogeni andrebbero ricercati, sia tra gli agenti batterici (anche se soprattutto sulla base di dettagliate informazioni specifiche al riguardo) pur con risorse diagnostiche adeguate, sia tra gli agenti virali, e ci riferiamo soprattutto ai nuovi (o rinnovati) agenti virali, quali ad esempio i Norovirus (26).

L'attenta osservazione microscopica di campioni significativi e in tempi reali sarà inoltre fattore importante per potere procedere razionalmente 
alle esecuzioni di colorazioni, soprattutto permanenti, per verificare o escludere la presenza di protozoi importanti e tutt'altro che sottostimabili, quali $D$. fragilis, da noi non rara, e i coccidi (Cryptosporidium spp. in particolare) da noi invece rarissimo se non assente (e così in tale indagine) ma sempre importante quanto meno nella diarrea del viaggiatore (e in diarree prolungate nel tempo), al pari evidentemente di altri patogeni batterici $(1,9)$.

Crediamo che un siffatto iter operativo metodologico sia garante di qualità e di economia gestionale, in altri termini di reale e razionale, perché ragionata, bontà diagnostica $(3,4,6)$

Tabella I. Risultati complessivi (anno 2004).

\begin{tabular}{|c|c|c|c|c|c|c|}
\hline \multicolumn{7}{|l|}{ AGENTE } \\
\hline \multirow{2}{*}{$\begin{array}{l}\text { EZIOLOGICO } \\
\text { Campylobacter }\end{array}$} & \multicolumn{2}{|c|}{ BAMBINI } & \multicolumn{2}{|c|}{ ADULTI } & \multicolumn{2}{|c|}{ TOTALI } \\
\hline & 36 & $14.2 \%$ & 21 & $8.0 \%$ & 57 & $11.0 \%$ \\
\hline Salmonella & 15 & $6.1 \%$ & 13 & $4.9 \%$ & 28 & $5.4 \%$ \\
\hline Shigella & 0 & - & 2 & $0.8 \%$ & 2 & $0.4 \%$ \\
\hline Vibrio & 0 & - & 3 & $1.1 \%$ & 3 & $0.6 \%$ \\
\hline Aeromonas & $\mathrm{I}$ & $0.4 \%$ & 1 & $0.4 \%$ & 2 & $0.4 \%$ \\
\hline Tossina A - C. difficile & 3 & $1.2 \%$ & 8 & $3.0 \%$ & II & $2.2 \%$ \\
\hline Rotavirus & 31 & $12.5 \%$ & 2 & $0.8 \%$ & 33 & $6.4 \%$ \\
\hline Adenovirus & 3 & $1.2 \%$ & 0 & - & 3 & $0.6 \%$ \\
\hline PROTOZOI & 3 & $1.2 \%$ & 10 & $3.7 \%$ & 13 & $2.5 \%$ \\
\hline POSITIVI TOTALI & 91 & $36.8 \%$ & 60 & $22.7 \%$ & $|5|$ & $29.5 \%$ \\
\hline NEGATIVI TOTALI & 156 & $63.2 \%$ & 204 & $77.3 \%$ & 360 & $70.5 \%$ \\
\hline Totale dei campioni & 247 & $100 \%$ & 264 & $100 \%$ & 511 & $100 \%$ \\
\hline
\end{tabular}

Tabella 2. Risultati relativi alle diarree acute.

\begin{tabular}{|c|c|c|c|c|c|c|}
\hline \multicolumn{7}{|l|}{ AGENTE } \\
\hline \multirow{2}{*}{$\begin{array}{l}\text { EZIOLOGICO } \\
\text { Campylobacter }\end{array}$} & \multicolumn{2}{|c|}{ BAMBINI } & \multicolumn{2}{|c|}{ ADULTI } & \multicolumn{2}{|c|}{ TOTALI } \\
\hline & 30 & $14.4 \%$ & 18 & $10.0 \%$ & 48 & $12.3 \%$ \\
\hline Salmonella & 11 & $5.3 \%$ & 12 & $6.7 \%$ & 23 & $5.9 \%$ \\
\hline Shigella & 0 & - & 2 & $1.1 \%$ & 2 & $0.5 \%$ \\
\hline Vibrio & 0 & - & 3 & $1.7 \%$ & 3 & $0.8 \%$ \\
\hline Aeromonas & $\mathrm{I}$ & $0.5 \%$ & 1 & $0.6 \%$ & 2 & $0.5 \%$ \\
\hline Tossina A - C. difficile & 3 & $1.4 \%$ & 6 & $3.3 \%$ & 9 & $2.3 \%$ \\
\hline Rotavirus & 31 & $14.8 \%$ & 2 & $1.1 \%$ & 33 & $8.5 \%$ \\
\hline Adenovirus & 3 & $1.4 \%$ & 0 & - & 3 & $0.8 \%$ \\
\hline PROTOZOI & 1 & $0.5 \%$ & 6 & $3.3 \%$ & 7 & $1.8 \%$ \\
\hline POSITIVI TOTALI & 80 & $38.3 \%$ & 50 & $27.8 \%$ & 130 & $33.4 \%$ \\
\hline NEGATIVI TOTALI & 129 & $61.7 \%$ & 130 & $72.2 \%$ & 259 & $66.6 \%$ \\
\hline Totale dei campioni & 209 & $100 \%$ & 180 & $100 \%$ & 389 & $100 \%$ \\
\hline
\end{tabular}

Tabella 3. Risultati relativi alle diarree protratte (Rotavirus eAdenovirus: non ricercati).

\begin{tabular}{lcccccr}
\hline $\begin{array}{l}\text { AGENTE } \\
\text { EZIOLOGICO }\end{array}$ & BAMBINI & \multicolumn{2}{c}{ ADULTI } & \multicolumn{2}{c}{ TOTALI } \\
\hline Campylobacter & 6 & $13.1 \%$ & 3 & $3.5 \%$ & 9 & $6.6 \%$ \\
\hline Salmonella & 4 & $10.5 \%$ & 1 & $1.2 \%$ & 5 & $4.1 \%$ \\
\hline Tossina A - C. difficile & 0 & - & 2 & $2.4 \%$ & 2 & $1.6 \%$ \\
\hline PROTOZOI & 2 & $5.3 \%$ & 4 & $4.8 \%$ & 6 & $4.9 \%$ \\
\hline POSITIVI TOTALI & $1 \mathrm{I}$ & $28.9 \%$ & 10 & $11.9 \%$ & 21 & $17.2 \%$ \\
\hline NEGATIVI TOTALI & 27 & $71.1 \%$ & 74 & $88.1 \%$ & 101 & $82.8 \%$ \\
\hline Totale dei campioni & 38 & $100 \%$ & 84 & $100 \%$ & 122 & $100 \%$ \\
\hline NB: I associazione tra Campylobacter e Salmonella & & &
\end{tabular}

Tabella 4. Infezioni intestinali complessive: distribuzione delle eziologie in rapporto al sesso.

\begin{tabular}{lccccrr}
\hline AGENTE & \multicolumn{2}{c}{ BAMBINI } & \multicolumn{2}{c}{ ADULTI } & \multicolumn{2}{c}{ TOTALI } \\
EZIOLOGICO & Maschi & Femm. & Maschi & Femm. & Maschi & Femm. \\
\hline Campylobacter & 21 & 14 & 15 & 6 & 36 & 20 \\
& & & & & $64.3 \%$ & $35.7 \%$ \\
\hline Salmonella & 8 & 6 & 6 & 7 & 14 & 13 \\
& & & & & $51.8 \%$ & $48.2 \%$ \\
\hline Rotavirus & 20 & II & I & I & 21 & 12 \\
& & & & & $63.6 \%$ & $36.4 \%$ \\
\hline PROTOZOI & I & 2 & 4 & 6 & 5 & 8 \\
& & & & & $38.5 \%$ & $61.5 \%$ \\
\hline
\end{tabular}

Tabella 5. Stagionalità ed eziologie osservate

\begin{tabular}{lrrrrrrr}
\hline & Campylobacter & \multicolumn{2}{c}{ Salmonella } & \multicolumn{2}{c}{ Rotavirus } \\
\hline Gennaio - Febbraio & 2 & $3.5 \%$ & 6 & $21.4 \%$ & 15 & $48.4 \%$ \\
\hline Marzo - Aprile & 7 & $12.3 \%$ & 4 & $14.3 \%$ & 11 & $35.5 \%$ \\
\hline Maggio - Giugno & 13 & $22.8 \%$ & 3 & $10.7 \%$ & 3 & $9.7 \%$ \\
\hline Luglio - Agosto & 12 & $21.1 \%$ & 9 & $32.1 \%$ & 0 & - \\
\hline Settembre - Ottobre & 15 & $26.3 \%$ & 4 & $14.3 \%$ & 2 & $6.4 \%$ \\
\hline Novembre - Dicembre & 8 & $14.0 \%$ & 2 & $7.2 \%$ & 0 & - \\
\hline Gennaio - Aprile & 9 & $15.8 \%$ & 10 & $35.7 \%$ & 26 & $83.9 \%$ \\
\hline Maggio - Agosto & 25 & $43.9 \%$ & 12 & $42.9 \%$ & 3 & $9.7 \%$ \\
\hline Settembre - Dicembre & 23 & $40.3 \%$ & 6 & $21.4 \%$ & 2 & $6.4 \%$ \\
\hline
\end{tabular}

Tabella 6. Distribuzione delle protozoosi

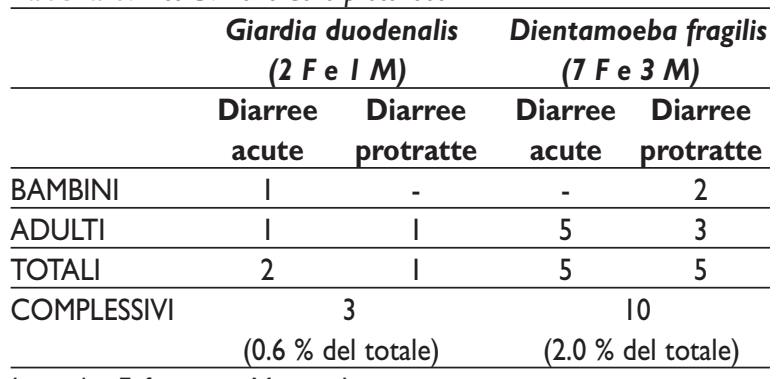

Legenda: F: femmina; M: maschio

\section{BIBLIOGRAFIA}

1. Bernieri F, Crotti D. Infezioni da protozoi. Microbiol Med 2001; 16: 98-108

2. Carattoli A, Ricci A. Infezioni da Salmonella. Microbiol Med 2001; 16: 33-36

3. Covelli I, Cuniato V, Cocco MP, Crotti D. Manuale pratico di diagnostica microbiologica. Associazione di volontariato J. E. Masslo Editore, Castelvolturno (CE), 2006, pagg. 149-159

4. Crotti D. Aspetti attuali nella diagnosi delle infezioni intestinali. La coprocoltura in chiave moderna. Le Monografie di Documenta, Edizioni Scientifiche Mascia Brunelli - Biolife Editore, Milano, 1997, pagg. 18-20

5. Crotti D. Campylobacteriosi nel perugino nel primo biennio del nuovo secolo: alcuni aspetti e considerazioni di microbiologia clinica. Microbiol Med 2004; 19: $37-41$

6. Crotti D. L'esame microbiologico dei campioni di feci: procedure operative standard. In: Cristallo A. F. La Medicina di Laboratorio nella Pratica medica. Volume di aggiornamento e ampliamento. Selecta 
Medica Editore, 2006, pagg. 129-138

7. Crotti D, D’Annibale ML. Diagnostica ed epidemiologia delle infezioni intestinali umane nel territorio perugino nel corso del 2003. BML news 2004; 10: 7-15

8. Crotti D. D'Annibale ML. Enteriti da Campylobacter e generi correlati: quale endemia? Brevi considerazioni. GIMMOC 2005; Vol. IX, $\mathrm{N}^{\circ} 2$, 122-125

9. Crotti D, D’Annibale ML, Fonzo G, Lalle M, Cacciò SM, Pozio E. Dientamoeba fragilis is more prevalent than Giardia duodenalis in children and adults attending a day care centre in Central Italy. Parasite 2005; 12: $165-170$

10. Crotti D, D’Annibale ML, Fonzo G, Medori MC, Ubaldi M. Diarree acute e protratte nella popolazione del territorio perugino: diagnosi microbiologica ed aspetti clinico-epidemiologici relativi al 2001. Le Infezioni in Medicina 2002; 2: 81-87

11. Crotti D, D’Annibale ML, Medori MC. Diagnostica microbiologica ed epidemiologia locale delle enteriti acute e protratte: risultati relativi all'anno 2002. Giorn It Mal Inf 2003; 9: 205-212

12. Crotti D, D'Annibale ML, Spinelli M et al. Antibiotico-rsistenza in Campylobacter jejuni/coli in Italia nel corso del 2002: risultati di una indagine policentrica. Microbiol Med 2003; 18: 9-14

13. Crotti D, Luzzi I. Infezioni da Yesinia enterocolitica. Microbiol Med 2001; 16: 33-36

14. Crotti D, Luzzi I. Infezioni da Campylobacter. Microbiol Med 2001; 16: 37-42

15. Crotti D, Medori MC, D’Annibale ML, Fonzo G, Del Sante M. Infezione da Clostridium difficile in pazienti con diarrea nosocomiale e acquisita in comunità a Perugia. Microbiol Med 2001; 16: 313-315

16. Crotti D, Medori MC, Fonzo G, Del Sante M, D'Annibale ML. Diarree in età pediatrica: l'infezione, la diagnosi, l'eziologia. Microbiol Med 1999; 14: 263-268

17. Dionisi AM, Crotti D, Pezzetti G et al. CAMPYG: sorveglianza delle infezioni da Campylobacter in Italia. Microbiol Med 2002; 17: 58-63

18. Giammanco A, Amato T, Morabito S, Caprioli A. Infezioni da ceppi patogeni di Escherichia coli. Microbiol Med 2001; 16: 25-32

19. Luzzi I, Dionisi AM. Infezioni da Shigella. Microbiol Med 2001; 16: 22-24

20. Mastrantonio P, Cerquetti M. Infezioni da Clostridium difficile. Microbiol Med 2001; 16: 61-65

21. Medici MC, Arcangeletti MC, Martinelli M, Valcavi P, Chezzi C, Dettori G. Infezioni da Rotavirus. Microbiol Med 2001; 16: 78-91

22. Piersimoni C, Ripabelli G. Infezioni da Aeromonas e Plesiomonas. Microbiol Med 2001; 16: 52-60

23. Podda R, Porcu PP, Arese MG, Crotti D. Enterite acuta da Yersinia enterocolitica in un bambino talassemico: descrizione del caso e considerazioni generali. Microbiol Med 2000; 15: 343-345

24. Ripabelli G, Luzzi I. Infezioni da batteri del genere Vibrio. Microbiol Med 2001; 16: 43-51

25. Rossi S, Crotti D. Enterite acuta autoctona da Plesiomonas shigelloides. Microbiol Med 1999; 14:
341-343

26. Ruggeri FM, Colone M, Monini M, Iosi F. Infezioni da virus del gruppo di Norwalk. Microbiol Med 2001; 16: $92-97$

27. Torres ME, Pirez MC, Schelotto F et al. Etiology of Children's Diarrhea in Montevideo, Uruguay : Associated Pathogens and Unusual Isolates. JMC 2001; 39: 2134-2139

\section{Daniele Crotti}

Strada Comunale per Pilonico Paterno 4 06080 Pianello, Perugia

Tel.: 075602372

E-mail: nenedc@tin.it 